\title{
Sentinel lymph node mapping in high-risk endometrial cancer: a systematic review and meta-analysis
}

\author{
Qiang Ji, Xiuying Wang, Jiyong Jiang, Liyan Chen \\ Gynecological Oncology Ward, Dalian Maternal and Child Health Hospital Affiliated to Dalian Medical University, Dalian, China \\ Contributions: (I) Conception and design: Q Ji; (II) Administrative support: J Jiang; (III) Provision of study materials or patients: L Chen; (IV) \\ Collection and assembly of data: Q Ji; (V) Data analysis and interpretation: X Wang; (VI) Manuscript writing: All authors; (VII) Final approval of \\ manuscript: All authors. \\ Correspondence to: Xiuying Wang. Dalian Maternal and Child Health Hospital Affiliated to Dalian Medical University, Dalian, China. \\ Email: wangxiuying_1972@163.com.
}

\begin{abstract}
Background: In the staging of endometrial cancer (EC), the role of sentinel lymph node (SLN) mapping for high-risk EC is still unclear.

Methods: Two authors independently reviewed abstracts and full-text articles for inclusion and assessed study quality. English studies published in PubMed, Embase, and Cochrane Library before 20th SEP, 2019 were retrieved to perform a systematic evaluation and meta-analysis which evaluate the detection rate and diagnostic accuracy of SLN mapping in high-risk EC. Statistical analysis was conducted using stata14.0 software.

Results: A total of 12 studies were included, including 758 high-risk EC patients. The detection rate of SLN mapping was $84.8 \%$ (95\% CI, 79.9-89.6\%). The pooled bilateral detection rate was $67.0 \%$ (95\% CI, $56.8-77.3 \%$ ). The pooled para-aortic detection rate was $8.4 \%$ (95\% CI, 1.8-14.9\%). The pooled sensitivity was $87 \%$ (95\% CI, 79-92\%), and the pooled specificity was 98\% (95\% CI, 96-99\%). Pooled negative predictive value (NPV) was 97.7\% (95\% CI, 96.4-99.1\%), AUC =0.99 (95\% CI, 0.97-0.099).

Conclusions: SLN mapping still has a high detection rate and diagnostic accuracy in high-risk EC. SLN mapping is a reliable alternative to systematic lymph node dissection, but its prognostic effect on high-risk EC is yet to be further studied and verified by large sample studies.
\end{abstract}

Keywords: Endometrial cancer (EC); sentinel lymph node (SLN); high-risk

Submitted Sep 30, 2020. Accepted for publication Dec 16, 2020.

doi: $10.21037 /$ gs-20-807

View this article at: http://dx.doi.org/10.21037/gs-20-807

\section{Introduction}

Endometrial cancer (EC) is one of the three major malignant tumors of the female reproductive tract. It was performed in the surgery and pathology stage in 1988 and developed for more than 30 years (1). The present staging system includes tumor grade, depth of muscular invasion, local and regional spread, lymph node metastasis, and distant metastasis, which lead to correct guiding significance for further postoperative treatment and prognosis of patients. Lymph node metastasis is the main route of EC metastasis, and lymph node metastasis or not has a significant impact on follow-up treatment and prognosis. If pelvic or abdominal aortic lymph node metastasis, the 5 -year survival rate is only $44-52 \%$ (2). At present, the status of the lymph nodes has been assessed by systematic lymphadenectomy. However, the problem is that the early endometrial carcinoma metastasis rate is low; systematic lymphadenectomy is more damaging to patients and has the risk of various long-term complications. However, current studies have suggested it did not significantly improve prognosis, and a multicenter randomized controlled trial at ASTEC has suggested that pelvic lymphadenectomy did not significantly improve both tumor-free and overall survival 
(OS) (3), the benefits of systematic lymphadenectomy were merely an evaluation of lymph node status. Therefore, clinicians need new methods to assess the status of lymph nodes without being so aggressive.

Sentinel lymph nodes (SLNs) was first identified by Cabanas (4) in 1977 as the first site of lymph node metastasis, reflecting the whole state of lymphatic metastasis. A negative SLN means that the remaining lymph nodes are not metastasized, so the SLN identification technology can reduce the trauma of lymph node surgery and reduce the occurrence of long-term complications. It is now used to treat breast cancer and melanoma. According to the risk of lymph node metastasis, it can be divided into low-risk and high-risk EC. At present, there is no unified standard for EC risk classification. The most commonly used method is "Mayo standard", which defines lowrisk endometrial carcinoma as histological grade G1/G2, myometrial invasion depth $\leq 50 \%$, and tumor diameter $\leq 2 \mathrm{~cm}$. High-risk EC includes risk factors, including G3, deep muscular infiltration, and special pathological types. According to the report, the rate of pelvic lymph node metastasis in low-risk EC was about $1.4-3 \%$, while in high-risk EC, it was about $6.4-23 \%$ (5). The National Comprehensive Cancer Network (NCCN) guidelines have approved sentinel node localization as a staging technique for $\mathrm{EC}$, as grade $2 \mathrm{~B}$ evidence. Prospective and retrospective clinical studies have shown that sentinel lymph node mapping (SLNM) combined with pathologic ultra-staging has satisfactory detection rate, sensitivity and negative predictive value (NPV) of SLN in patients with early lowrisk endometrial carcinoma, and does not affect progression free survival (PFS) and OS. However, the effect of SLN mapping in high-risk EC patients remains unclear. In the earlier version, NCCN guidelines suggested SLN mapping should be cautious for high-risk EC patients. In the latest version, it is believed that SLN mapping may also have a better effect in high-risk EC (6). However, the existing evidence is mostly retrospective studies, with few patients included in individual studies, and some studies hold different views (7). Therefore, it is necessary to evaluate existing evidence systematically. Different from the previous meta-analysis which did not clearly distinguish the risk types of patients, this study aims to include the study evaluating the diagnostic value of SLN mapping in high-risk EC. The main aim is to analyze the detection rate, bilateral detection rate, sensitivity, and specificity of SLN mapping in high-risk $\mathrm{EC}$, to provide a clinical reference.

We present the following article in accordance with the
PRISMA reporting checklist (available at http://dx.doi. org/10.21037/gs-20-807).

\section{Methods}

\section{Retrieval strategy}

PubMed, Embase, and Cochrane Library were searched. The last search was conducted on September 20, 2019. Search keywords: "Sentinel lymph node" and "endometrial cancer". The specific retrieval scheme is shown in Table 1.

\section{Inclusion and exclusion criteria}

According to the established inclusion and exclusion criteria, the two evaluators screened the literature, read the title and abstract. Inclusion criteria: (I) the original study on SLN drawing of high-risk EC line; (II) SLN drawing of the high-risk group was included in the EC line study. Exclusion criteria: (I) studies with fewer than ten patients; (II) meeting abstracts, reviews, case reports, or editorials. After reading the full text, it included research on the detection rate, sensitivity, and other relevant indicators of the report, and reached a consensus through discussion or listening to the third-party opinions in divergence.

Major outcome indicators include sensitivity or specificity of high-risk EC SLN mapping, detection rate, bilateral detection rate, para-aortic detection rate, NPV, and summary receiver operating characteristic (SROC) curve.

\section{Quality evaluation}

According to Cochrane's QUADAS-2 scale, two researchers evaluated the quality and bias risk of the included diagnostic studies in four aspects (patient selection, diagnostic experiment, gold standard, follow-up). All items were evaluated as three grades: "yes", "no" or "unknown". The results were input into the software to make the quality evaluation chart of diagnostic study (Figure 1). Disagreements were reached through the original literature review and discussion.

\section{Data extraction}

Two reviewers extracted the data. Data include author, year of publication, study design, patient population, SLN technology, relevant outcome data, and quality assessment programs. For SLN technology, we extracted data related 


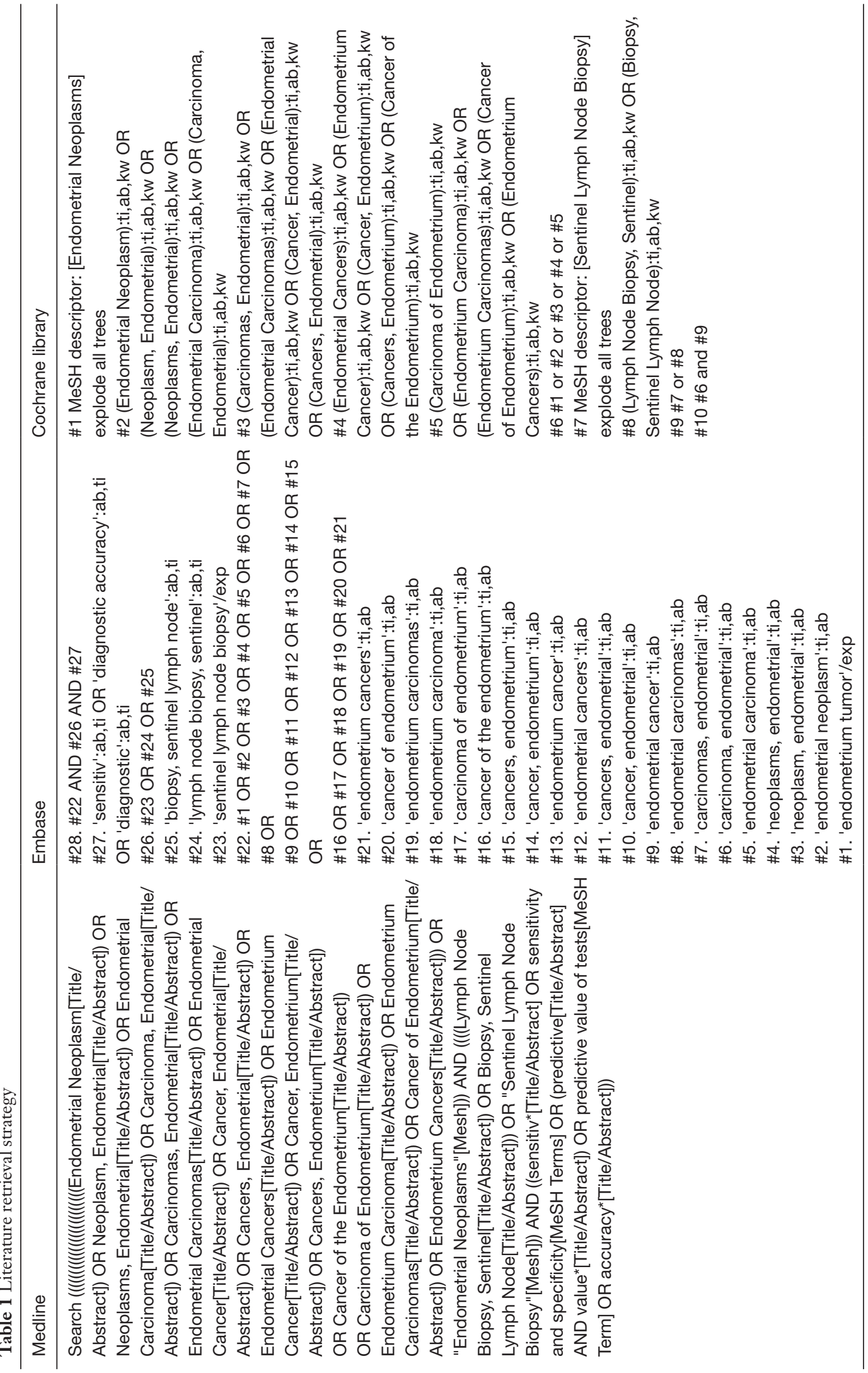




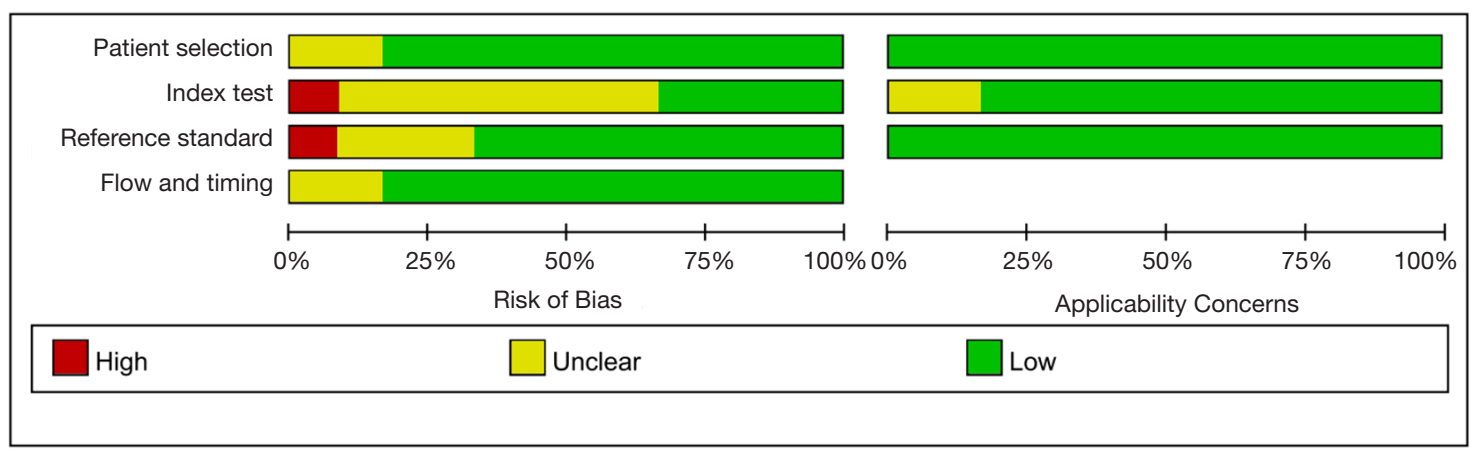

Figure 1 Quality assessment.

to surgical approach (robot, laparoscopic, laparotomy), injection site, tracer selection, and pathological evaluation. As for extracting and analyzing the data after the SLN algorithm included the pre-sln/post-sln algorithm.

\section{Statistical analysis}

We used stata14.0 for meta-analysis of the included trial data. The statistical model was selected according to the size of heterogeneity $\left(\mathrm{I}^{2} \geq 50 \%\right.$ random effect model, $\mathrm{I}^{2}<50 \%$ fixed effect model) for the detection rate, bilateral detection rate, and NPV meta-analysis. For sensitivity and specificity, we used a bivariate mixed effect model. Meta-regression was used to find the heterogeneity of major outcome indicators, and a funnel plot was used to detect publication bias between studies. All the above studies showed statistically significant differences at $\mathrm{P}<0.05$.

\section{Results}

After preliminary screening and full-text review, 12 studies were included in the final analysis, with the main objectives of evaluating detection rate and diagnostic accuracy (Figure 2). A total of 758 high-risk EC patients were included, and the baseline characteristics of the included studies are shown in Table 2. Retrospective studies accounted for the majority, with 7 (58.3\%) and 5 (41.7\%) prospective studies (Table 3). Eleven studies performed pelvic lymph node dissection with or without para-aortic lymph node dissection after removing the SLN. One study performed pelvic lymph node dissection and para-aortic lymph node dissection only after drawing failure. Eleven studies pooled the results of diagnostic accuracy, and seven studies pooled the detection rate.

\section{Detection rate}

A total of 7 studies could extract relevant data of detection rate of high-risk EC SLN mapping (514 patients), with the detection rate ranging from $73.2 \%$ to $100 \%$ and the pooled detection rate of $87.8 \%$ (95\% CI, $85.1-90.5 \%$ ). The bilateral detection rate was $55.6-90.5 \%$, and the pooled bilateral detection rate was $67.0 \%$ (95\% CI, 56.8-77.3\%). The para-aortic detection rate was $1.98-54.5 \%$ and the pooled para-aortic detection rate was $8.4 \%$ (95\% CI, $1.8-14.9 \%, 5$ studies) (Figure $3 A, B, C)(8-14)$.

\section{Diagnostic accuracy}

Eleven studies were conducted to evaluate the diagnostic accuracy of high-risk EC line SLN mapping. The pooled sensitivity was $87 \%$ (95\% CI, 79-92\%). The pooled specificity was $98 \%$ (95\% CI, 96-99\%). The pooled NPV was 97.7\% (95\% CI, 96.4-99.1\%) (Figure 4A,B) (7-9,11-18). The SROC curve is shown in Figure 5 , AUC $=0.99(95 \%$ CI, 0.97-0.99).

\section{Meta regression analysis and subgroup analysis}

In the combination of sensitivity analysis, there are a high heterogeneity $\mathrm{I}^{2}=56.36(26.90-85.77)$. Therefore, metaregression analysis was conducted on study design, whether the SLN algorithm was followed, super pathological staging, study scale, and tracer selection to find the source of heterogeneity. From the analysis results, the heterogeneity of sensitivity was mainly related to study design and ICG use, but not to follow SLN resection principle, pathological staging and study scale (Figure 6A). Subgroup analysis showed that retrospective study and non ICG tracer had higher sensitivity, 90\% (95\% CI, 84-96\%) and 90\% (95\% 


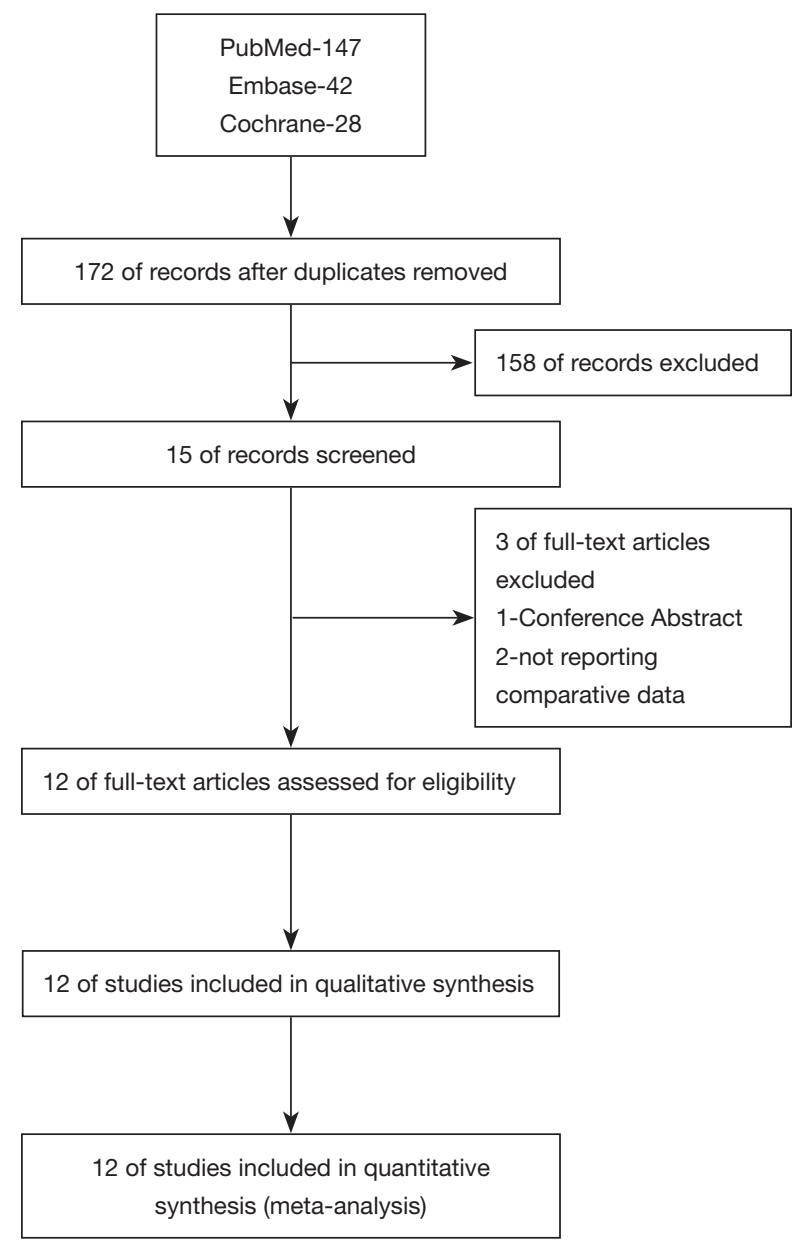

Figure 2 PRIMA flow chat.

CI, 83-98\%) respectively (Figure 6B).

\section{Sensitivity analysis}

Further sensitivity analysis (Figure 7) was carried out to explore the effect of single study before and after removal on pooled effect size and pooled sensitivity. The result showed that Ye 2019 (7) had a great effect on the pooled effect size, which may be the source of heterogeneity. After excluding this study, the pooled sensitivity was $89 \%$ (95\% CI, 84-93\%), and the heterogeneity was significantly improved $\left(\mathrm{I}^{2}=0.0 \%\right)$ (Figure 8$)$. It is considered that the heterogeneity may be derived from the prospective design of this study and the use of ICG, and the conclusion of this study was also contrary to the other studies.

\section{Publication bias}

The publication bias was analyzed and adjusted for 11 studies that evaluated the diagnostic accuracy. The result was shown in the Deek's funnel plot $(\mathrm{P}=0.01<0.1)$ (Figure 9). The pooled effect size of both the fixed-effect model and the random-effect model was 5.616 (95\% CI, 4.612-6.621) before the funnel plot was trimmed and filled, while that was changed to 191.369 (95\% CI, 78.606-465.895) after trimming and filling. The significant change in the pooled effect size suggested the possible publication bias.

\section{Discussion}

Clinical studies have shown that patients with early EC have a low incidence of abdominopelvic lymph node metastasis. 


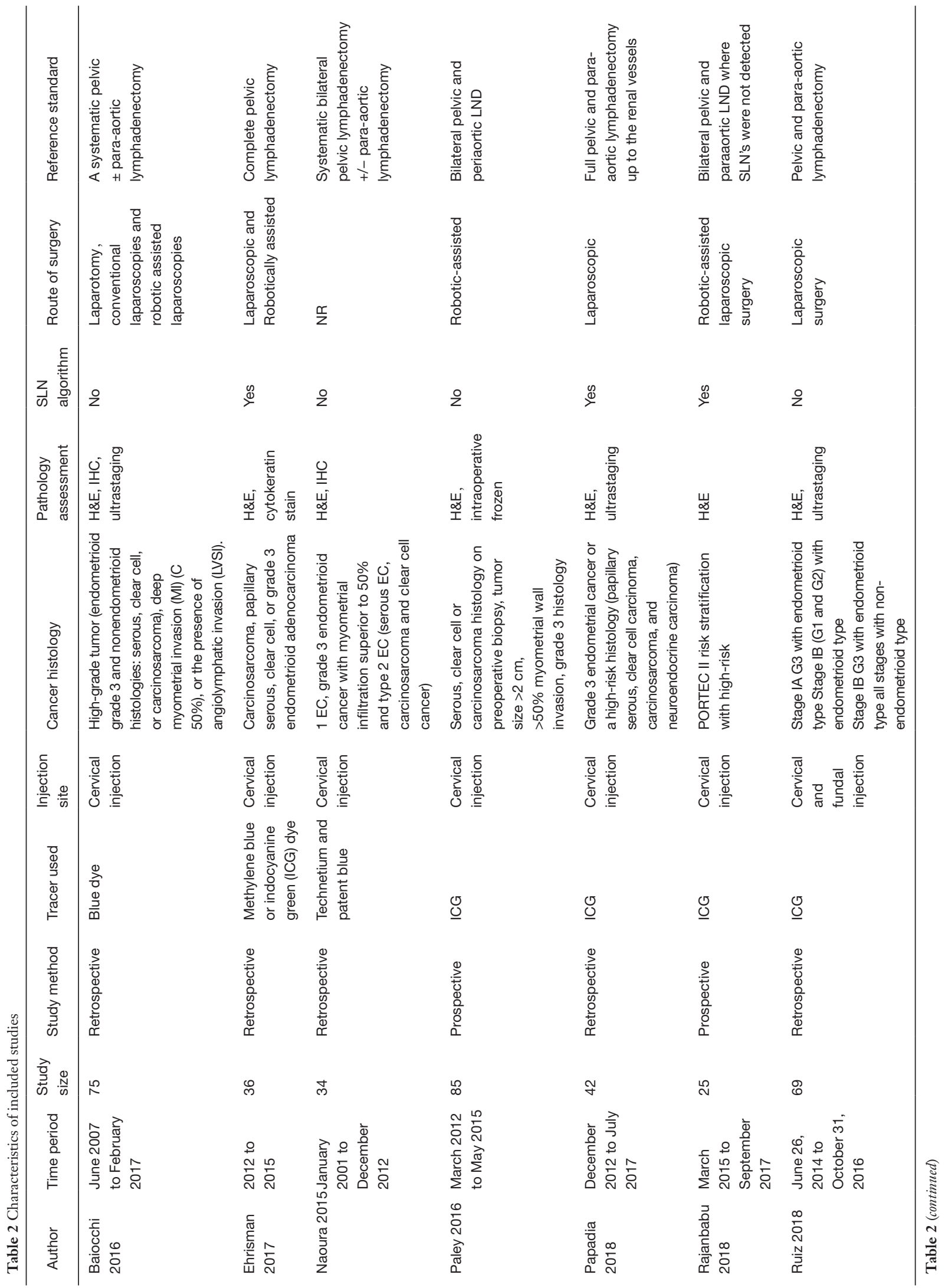




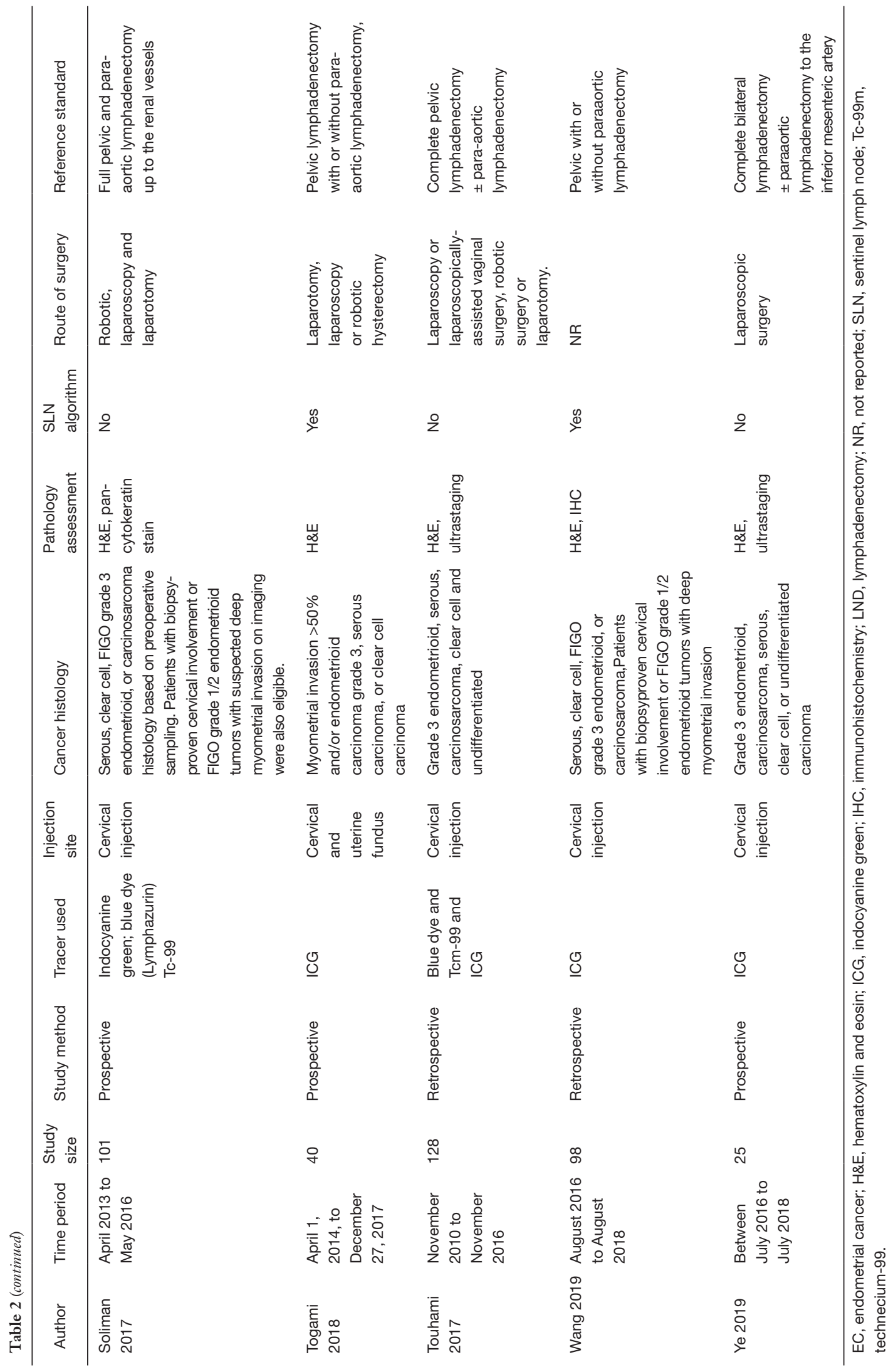




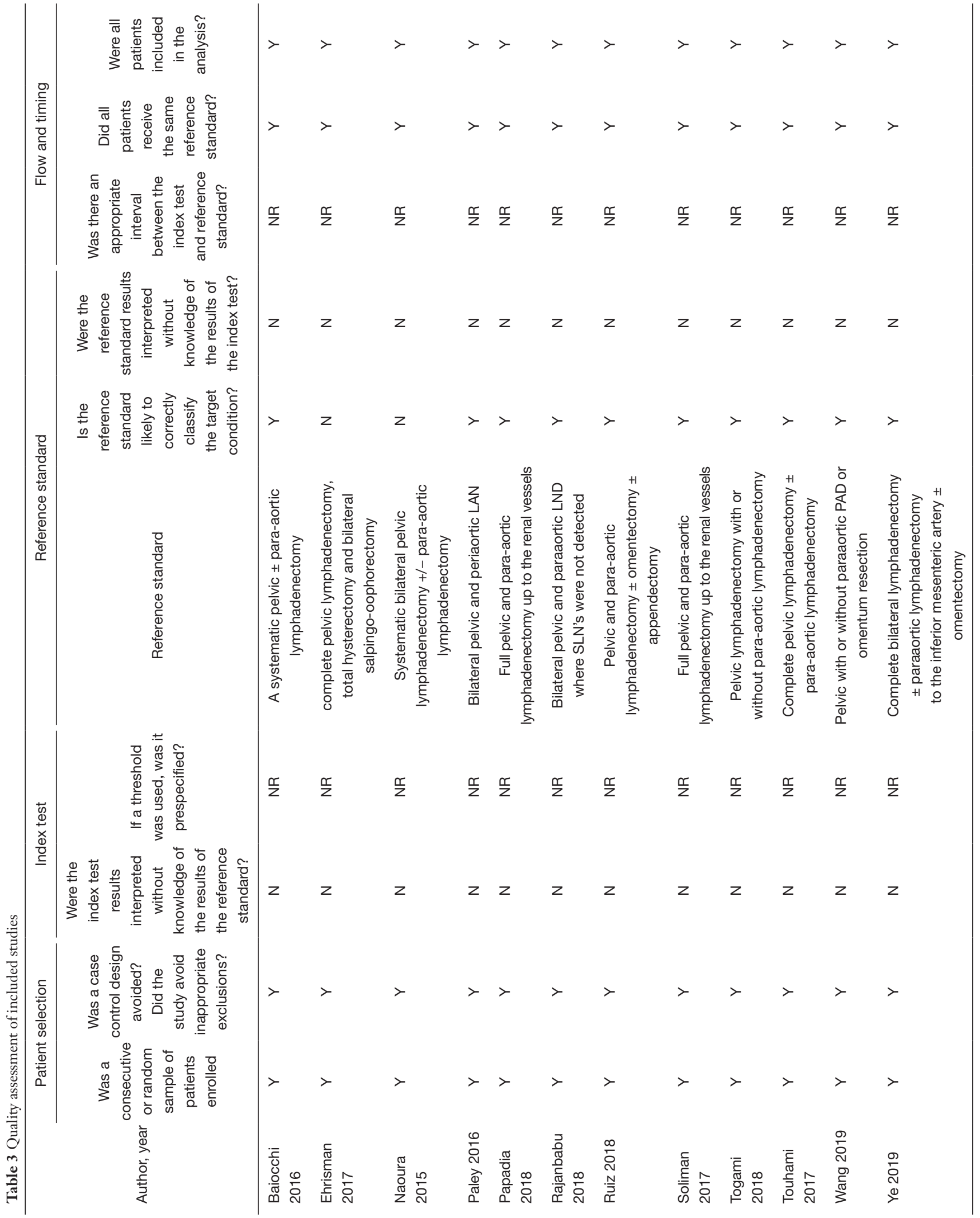


A

Study

ID

$\%$

ES $(95 \% \mathrm{Cl}) \quad$ Weight

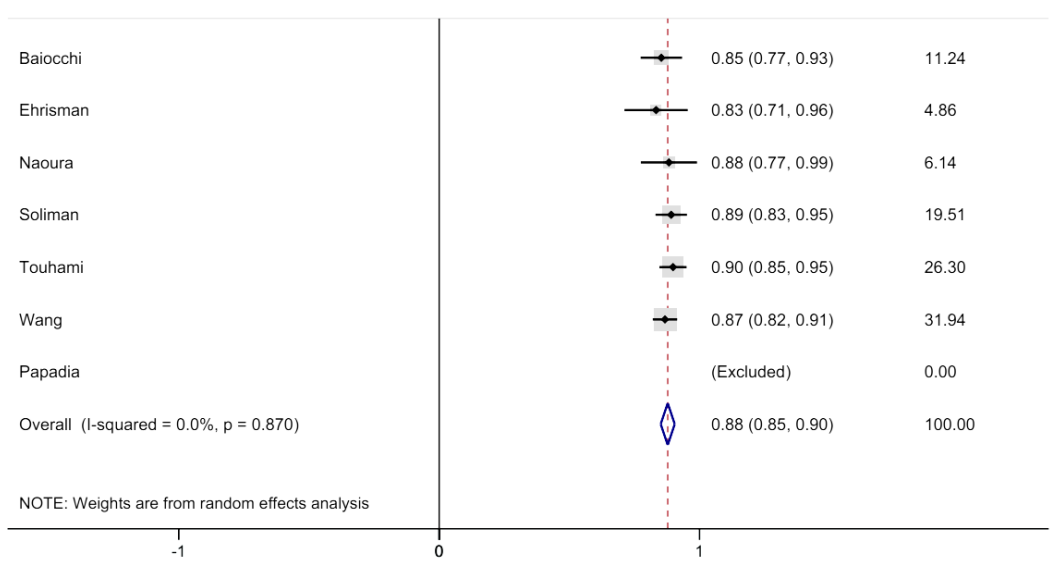

B
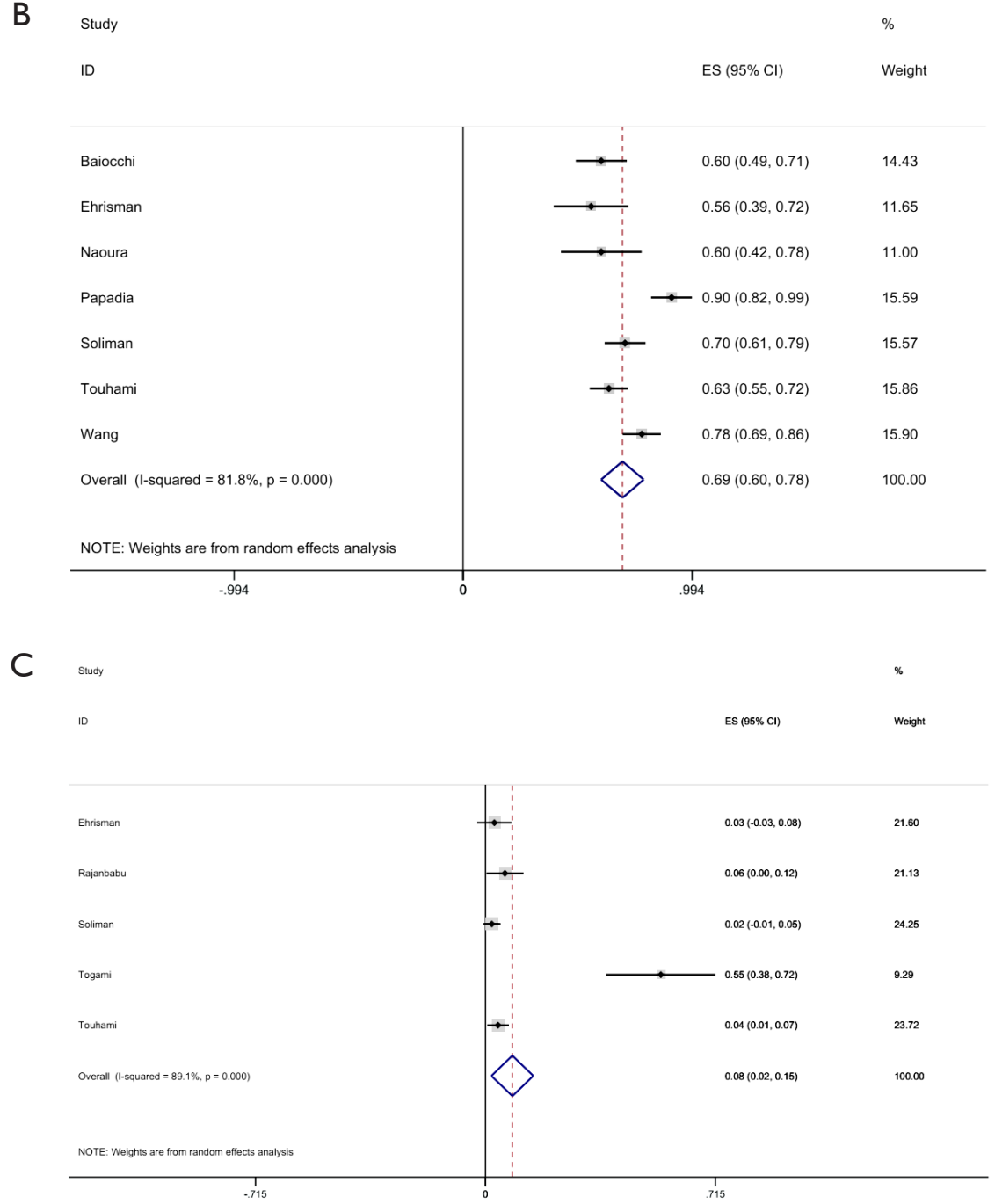

Figure 3 Sentinel lymph node (SLN) detection rate. (A) Detection rate; (B) bilateral detection rate; (C) para-aortic detection rate. 
A

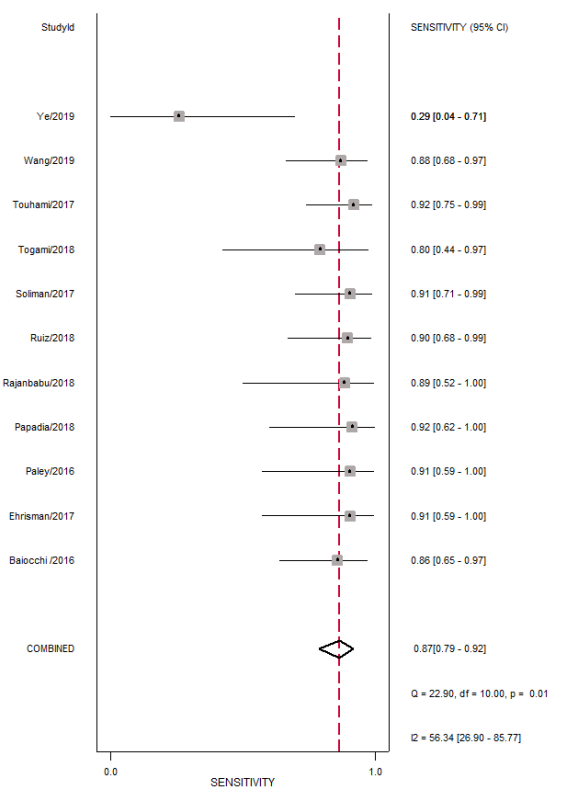

B

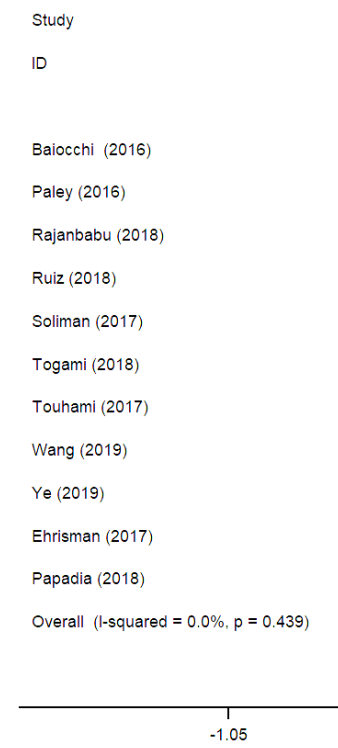

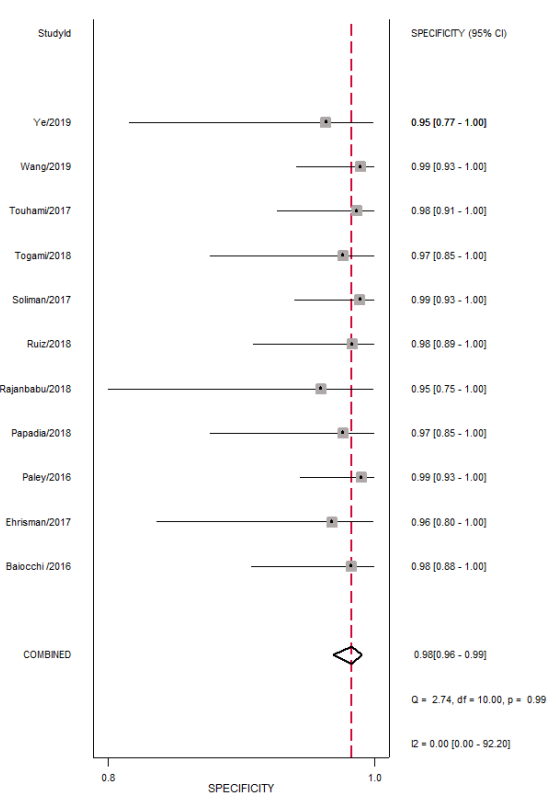

$\%$

ES $(95 \% \mathrm{Cl}) \quad$ Weight

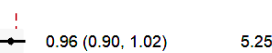

$0.96(0.90,1.02) \quad 5.25$

$+0.99(0.96,1.01) \quad 29.26$

$\because 0.95(0.85,1.05) \quad 2.00$

$0.96(0.90,1.01)$

\begin{tabular}{l}
$0.99(0.96,1.01) \quad 24.28$ \\
\hline
\end{tabular}

$\because 0.94(0.87,1.02) \quad 3.08$

\begin{tabular}{l}
$-0.98(0.95,1.02) \quad 16.25$ \\
\hline
\end{tabular}

$\rightarrow 0.97(0.94,1.01) \quad 13.36$

$\begin{array}{l:ll}\longrightarrow & 0.81(0.66,0.96) & 0.79\end{array}$

(Excluded) $\quad 0.00$

(Excluded) $\quad 0.00$

$0.98(0.96,0.99) \quad 100.00$

Figure 4 Sentinel lymph node (SLN) diagnostic accuracy. (A) Sensitivity and specificity; (B) negative predictive value (NPV).

Systematic lymphadenectomy can assess the lymph node status, but it will lead to neurovascular injury, lymphocyst, chronic lymphedema of the lower limb, rare chylous ascites and infection and other complications, seriously affecting the quality of life of patients. Moreover, lymph nodes are immune organs, and it is currently believed that lymph nodes that have not yet metastasized still have the defense function of blocking the spread of cancer cells. Therefore, systematic lymphadenectomy can weaken the anti-tumor immunity of the body while increase the potential risk of distant metastasis of occult cancer foci. The SLN mapping technique can avoid the complications caused by systematic lymphadenectomy to a great extent on the premise of ensuring the diagnostic accuracy. In addition, because patients with low-risk EC who meet the "Mayo criteria" have a lymph node metastasis rate or recurrence rate of less than $1 \%$ and can avoid systematic lymphadenectomy. Collectively, the application of SLN mapping technique in 


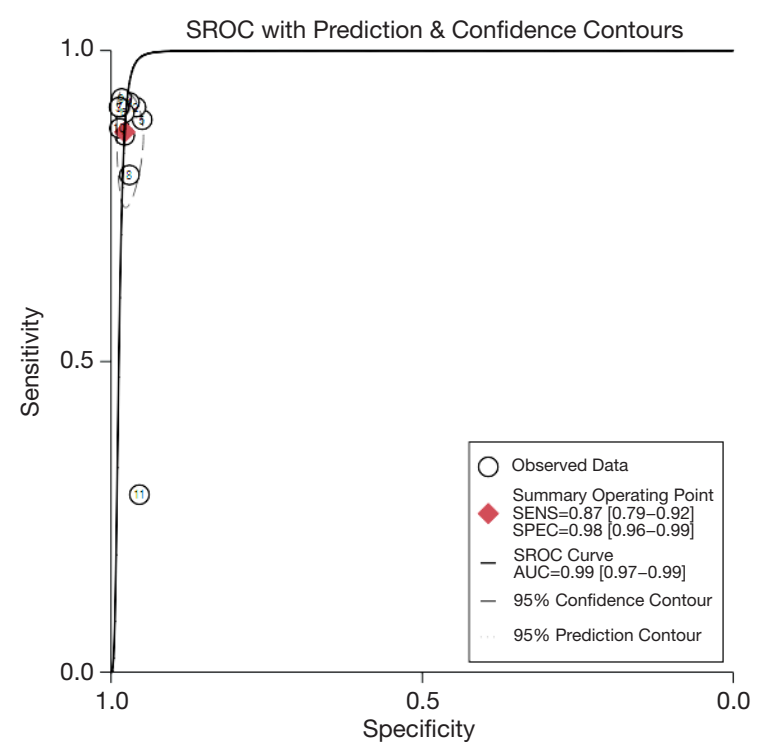

Figure 5 Summary receiver operating characteristic (SROC) curve.

patients with high-risk EC is of greater significance.

A total of 12 studies were included in this systematic evaluation and meta-analysis, including 758 high-risk EC patients, and the detection rate of SLN mapping was $84.8 \%$ (95\% CI, 79.9-89.6\%). The pooled bilateral detection rate was $67.0 \%$ (95\% CI, 56.8-77.3\%). The pooled para-aortic detection rate was $8.4 \%$ (95\% CI, 1.8-14.9\%). The pooled sensitivity was $87 \%$ (95\% CI, 79-92\%), and the pooled specificity was $98 \%$ (95\% CI, 96-99\%). Pooled NPV was 97.7\% (95\% CI, 96.4-99.1\%). Our analysis concluded that SLN mapping was still of sufficient diagnostic accuracy in high-risk EC patients and could be a reliable alternative to systematic lymptomies, which meant that patients with EC did not need to assess the risk of lymph node metastasis but could use SLN mapping to evaluate the status of lymph nodes. On a larger scale, EC patients would receive more accurate treatment and avoid unnecessary complications and pain.

The included studies' overall detection rate in this paper ranged from $73.2 \%$ to $100 \%$, and the bilateral detection rate was $55.6 \%$ to $90.5 \%$. The factors that may affect the detection rate of SLN have also been reported in previous studies. Patients' relevant characteristics include age, weight, menopause, pelvic surgery, history (cesarean section; accessory surgery; appendicitis. cervical surgery; hysteromyoma nucleus excises), etc. Tumor related characteristics include lymph node metastasis, histological type, tumor stage, tumor size, depth of muscle invasion, LVSI, etc. However, most of the relationships with detection rates are still unclear. In a recent study, the authors analyzed these factors and found that sentinel node detection was only associated with the injector (19). Khoury-Collado et al. (20) reported that higher detection rates and lower false-negative rate could be achieved through the learning curve of more than 30 cases. Simultaneously, the SLN algorithm proposed by Barlin et al. (21) can also reduce the false-negative rate and improve the diagnostic accuracy of SLN mapping, mainly including (I) the assessment of peritoneum and serosa, peritoneal washing fluid. (II) Retroperitoneal evaluation, which included a positive SLN and any lymph nodes with suspected metastasis, whether stained or not. (III) If SLN is not found on one side, perform lymph node dissection on one side (external iliac, internal iliac, and obturator foramen). After its retrospective application of the principle, the false-negative rate was reduced from $15 \%$ to $2 \%$, and the NPV was as high as $99.8 \%$. Therefore, for the injection link of SLN drawing, the tracer's injection process should be standardized, and the injection personnel should be trained accordingly. Simultaneously, the injection personnel should be fixed as much as possible. The application of the SLN algorithm can also reduce the false-negative rate of SLN drawing.

Some studies show that super pathological staging can detect an additional $4.5 \%$ of low-load metastases (22). However, tumor micrometastases and isolated tumor cells' clinical significance is still unclear, so patients with tumor micrometastases need adjuvant therapy and what benefits adjuvant therapy will bring. In a recent multicenter retrospective study conducted by Ignatov et al., 428 patients were included, and the results showed micrometastases were associated with reduced disease-free survival (DFS) in patients with EC, with a 2-fold increased risk of recurrence. Together, adjuvant therapy may improve DFS in patients with micrometastases, with a $71 \%$ reduced risk (23).

The 12 studies included in this study did not report the prognostic impact of SLN mapping on high-risk EC. Previous studies showed no significant difference in progression-free survival between SLN resection and systematic lymph node dissection (24-28), but the subjects were mostly low-risk EC. The effect of SLN mapping on the long-term prognosis of high-risk EC is still to be explored.

This study summarizes relevant studies on SLN mapping of high-risk EC lines given existing disputes, but there are 


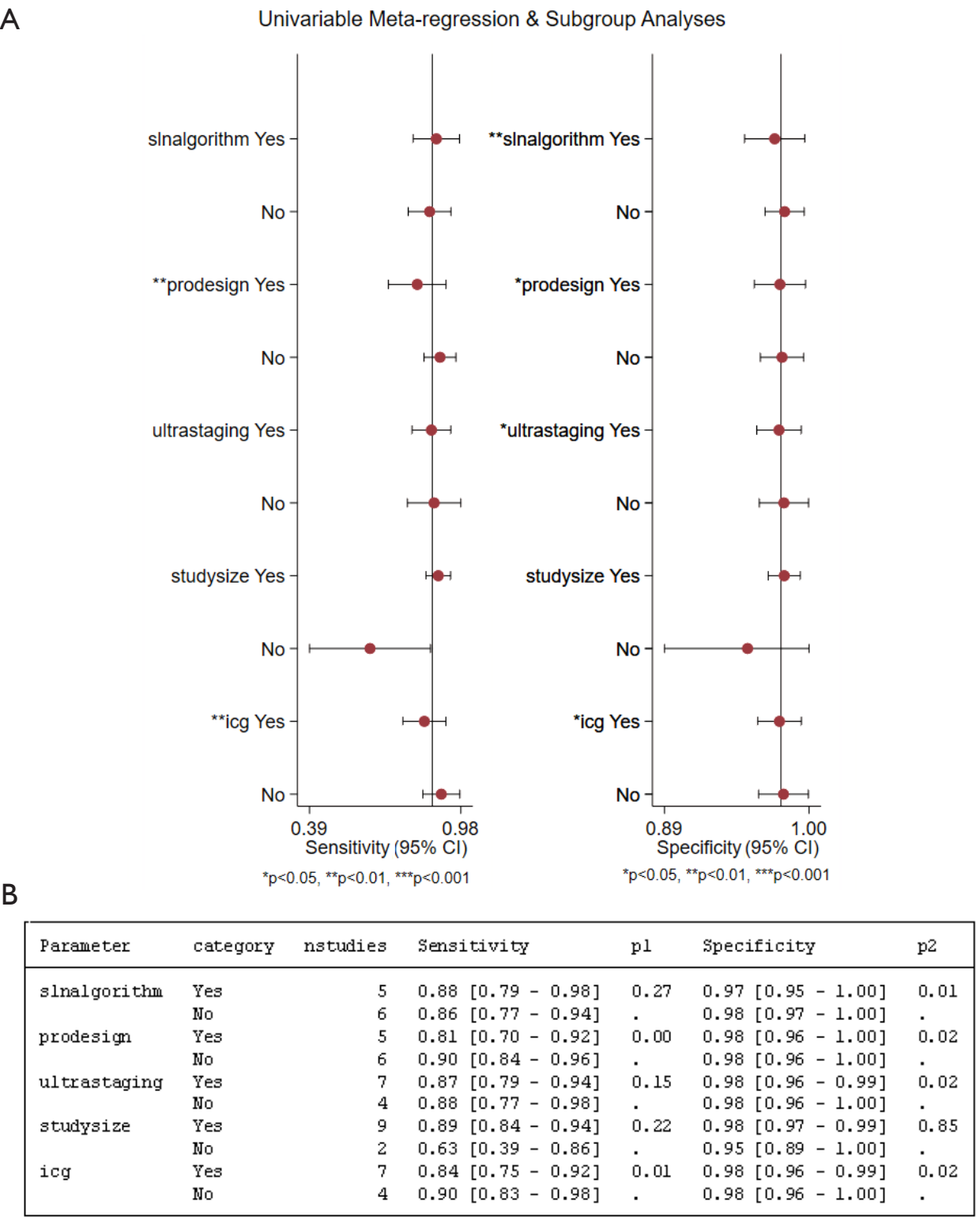

B

Figure 6 Meta regression analysis of sources of heterogeneity.

still some limitations. In this paper's studies, multiple tracers and injection routes have been used, affecting the actual effect of specific tracers and injection routes. Moreover, all studies are limited to English, and key studies may be omitted.

This study included 12 studies and conducted a systematic evaluation and meta-analysis. SLN mapping still has a high detection rate and diagnostic accuracy in highrisk EC. SLN mapping is a reliable alternative to systematic lymph node dissection, but its prognostic impact on highrisk EC has yet to be further studied and needs to be verified by large sample studies. 


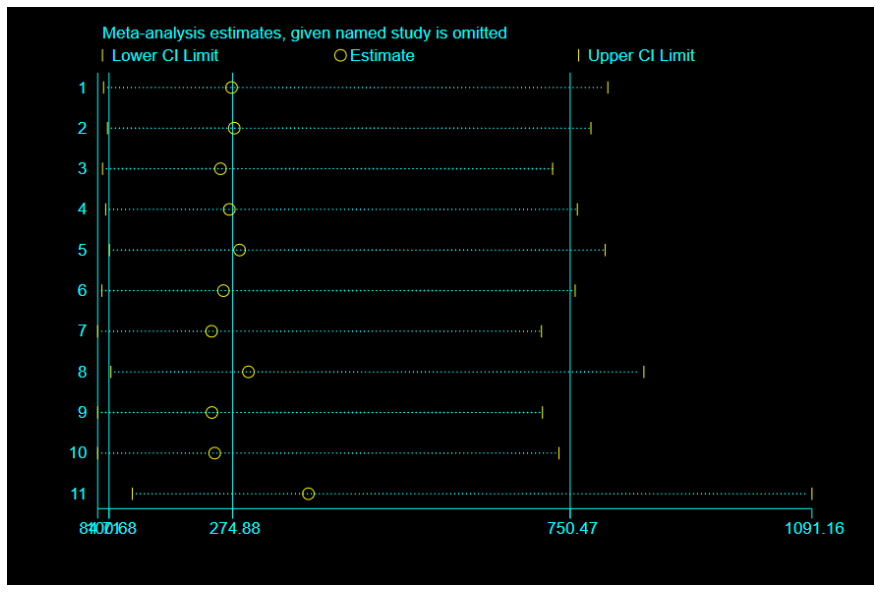

Figure 7 Sensitivity analysis.

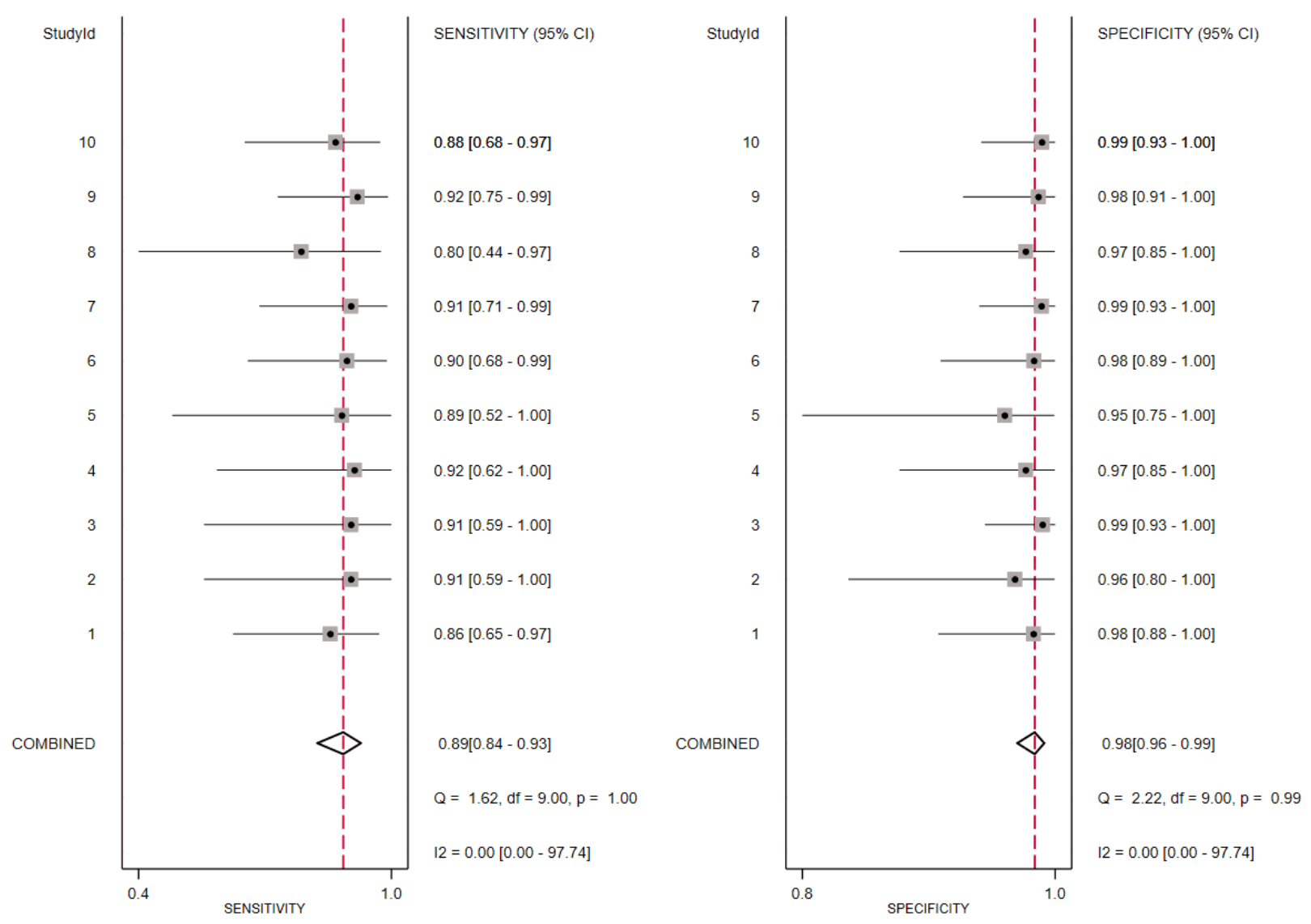

Figure 8 Sentinel lymph node (SLN) diagnostic accuracy sensitivity and specificity (exclude Ye L 2019). 


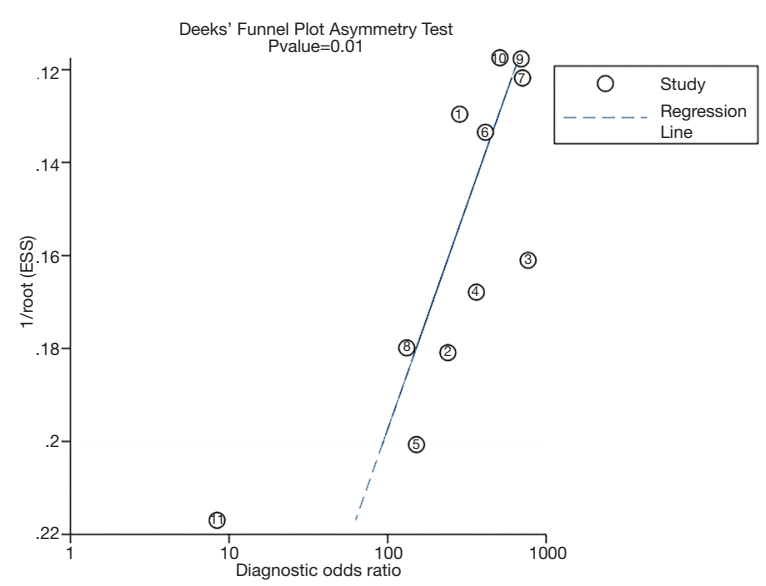

Figure 9 Publication bias.

\section{Acknowledgments}

Funding: None.

\section{Footnote}

Reporting Checklist: The authors have completed the PRISMA reporting checklist. Available at http://dx.doi. org/10.21037/gs-20-807

Conflicts of Interest: All authors have completed the ICMJE uniform disclosure form (available at http://dx.doi. org/10.21037/gs-20-807). The authors have no conflicts of interest to declare.

Ethical Statement: The authors are accountable for all aspects of the work in ensuring that questions related to the accuracy or integrity of any part of the work are appropriately investigated and resolved.

Open Access Statement: This is an Open Access article distributed in accordance with the Creative Commons Attribution-NonCommercial-NoDerivs 4.0 International License (CC BY-NC-ND 4.0), which permits the noncommercial replication and distribution of the article with the strict proviso that no changes or edits are made and the original work is properly cited (including links to both the formal publication through the relevant DOI and the license). See: https://creativecommons.org/licenses/by-nc-nd/4.0/.

\section{References}

1. Mikuta JJ. International Federation of Gynecology and
Obstetrics staging of endometrial cancer 1988. Cancer 1993;71:1460-3.

2. Bogani G, Ditto A, Chiappa V, Raspagliesi F. Sentinel node mapping in endometrial cancer. Transl Cancer Res 2019;8:2218-9.

3. Kitchener H, Swart AM, Qian Q, et al. Efficacy of systematic pelvic lymphadenectomy in endometrial cancer (MRC ASTEC trial): a randomised study. Lancet 2009;373:125-36.

4. Cabanas RM. An approach for the treatment of penile carcinoma. Cancer 1977;39:456-66.

5. Kumar S, Podratz KC, Bakkum-Gamez JN, et al. Prospective assessment of the prevalence of pelvic, paraaortic and high paraaortic lymph node metastasis in endometrial cancer. Gynecol Oncol 2014;132:38-43.

6. (NCCN) NCCN. Uterine Neoplasms, Clinical Practice Guidelines in Oncology. Version 1. 2019. Available online: http://www.nccn.org/professionals/physician_gls/pdf/ uterine.pdf. Accessed October 17, 2018.

7. Ye L, Li S, Lu W, et al. A Prospective Study of Sentinel Lymph Node Mapping for Endometrial Cancer: Is It Effective in High-Risk Subtypes? Oncologist 2019;24:e1381-7.

8. Baiocchi G, Mantoan H, Kumagai LY, et al. The Impact of Sentinel Node-Mapping in Staging High-Risk Endometrial Cancer. Ann Surg Oncol 2017;24:3981-7.

9. Ehrisman J, Secord AA, Berchuck A, et al. Performance of sentinel lymph node biopsy in high-risk endometrial cancer. Gynecol Oncol Rep 2016;17:69-71.

10. Naoura I, Canlorbe G, Bendifallah S, et al. Relevance of sentinel lymph node procedure for patients with high-risk endometrial cancer. Gynecol Oncol 2015;136:60-4.

11. Papadia A, Gasparri ML, Radan AP, et al. Retrospective validation of the laparoscopic ICG SLN mapping in patients with grade 3 endometrial cancer. J Cancer Res Clin Oncol 2018;144:1385-93.

12. Soliman PT, Westin SN, Dioun S, et al. A prospective validation study of sentinel lymph node mapping for highrisk endometrial cancer. Gynecol Oncol 2017;146:234-9.

13. Touhami O, Grégoire J, Renaud MC, et al. Performance of sentinel lymph node (SLN) mapping in high-risk endometrial cancer. Gynecol Oncol 2017;147:549-53.

14. Wang T, Hu Y, He Y, et al. A retrospective validation study of sentinel lymph node mapping for high-risk endometrial cancer. Arch Gynecol Obstet 2019;299:1429-35.

15. Togami S, Kawamura T, Fukuda M, et al. Prospective study of sentinel lymph node mapping for endometrial cancer. Int J Gynaecol Obstet 2018;143:313-8. 
16. Ruiz R, Gorostidi M, Jaunarena I, et al. Sentinel Node Biopsy in Endometrial Cancer With Dual Cervical and Fundal Indocyanine Green Injection. Int J Gynecol Cancer 2018;28:139-44.

17. Rajanbabu A, Agarwal R. A prospective evaluation of the sentinel node mapping algo-rithm in endometrial cancer and correlation of its performance against endometrial cancer risk subtypes. Eur J Obstet Gynecol Reprod Biol 2018;224:77-80.

18. Paley PJ, Veljovich DS, Press JZ, et al. A prospective investigation of fluorescence im-aging to detect sentinel lymph nodes at robotic-assisted endometrial cancer staging. Am J Obstet Gynecol 2016;215:117.e1-7.

19. Ianieri MM, Puppo A, Novelli A, et al. Sentinel Lymph Node Biopsy in the Treatment of Endometrial Cancer: Why We Fail? Results of a Prospective Multicenter Study on the Factors Associated with Failure of Node Mapping with Indocyanine Green. Gynecol Obstet Invest 2019;84:383-9.

20. Khoury-Collado F, Glaser GE, Zivanovic O, et al. Improving sentinel lymph node de-tection rates in endometrial cancer: how many cases are needed? Gynecol Oncol 2009; 115:453-5.

21. Barlin JN, Khoury-Collado F, Kim CH, et al. The importance of applying a sentinel lymph node mapping algorithm in endometrial cancer staging: beyond removal of blue nodes. Gynecol Oncol 2012;125:531-5.

22. Kim CH, Soslow RA, Park KJ, et al. Pathologic

Cite this article as: Ji Q, Wang X, Jiang J, Chen L. Sentinel lymph node mapping in high-risk endometrial cancer: a systematic review and meta-analysis. Gland Surg 2020;9(6):2091-2105. doi: 10.21037/gs-20-807 ultrastaging improves micrometastasis detection in sentinel lymph nodes during endometrial cancer staging. Int J Gynecol Cancer 2013;23:964-70.

23. Ignatov $A$, Lebius $C$, Ignatov $T$, et al. Lymph node micrometastases and outcome of endometrial cancer. Gynecol Oncol 2019;154:475-9.

24. How J, Gauthier C, Abitbol J, et al. Impact of sentinel lymph node mapping on recur-rence patterns in endometrial cancer. Gynecol Oncol 2017;144:503-9.

25. Raimond E, Ballester M, Hudry D, et al. Impact of sentinel lymph node biopsy on the therapeutic management of early-stage endometrial cancer: Results of a retrospective multicenter study. Gynecol Oncol 2014;133:506-11.

26. Schiavone MB, Zivanovic O, Zhou Q, et al. Survival of Patients with Uterine Carcinosarcoma Undergoing Sentinel Lymph Node Mapping. Ann Surg Oncol 2016;23:196-202.

27. Daraï E, Dubernard G, Bats AS, et al. Sentinel node biopsy for the management of early stage endometrial cancer: long-term results of the SENTI-ENDO study. Gynecol Oncol 2015;136:54-9.

28. Zahl Eriksson AG, Ducie J, Ali N, et al. Comparison of a sentinel lymph node and a selective lymphadenectomy algorithm in patients with endometrioid endometrial carcinoma and limited myometrial invasion. Gynecol Oncol 2016;140:394-9.

(English Language Editor: J. Chapnick) 